\title{
Objectively Measured Physical Activity of Vietnamese Adults With Type 2 Diabetes: Opportunities to Intervene
}

\author{
Vuong Van Do ${ }^{1}$, Jonine Jancey ${ }^{2}$, Ngoc Minh Pham ${ }^{2,3}$, Chung Thanh Nguyen ${ }^{4}$, Minh Van Hoang ${ }^{1}$, Andy H. Lee ${ }^{2}$ \\ ${ }^{1}$ Center for Population Health Sciences, Hanoi University of Public Health, Hanoi, Vietnam; ${ }^{2}$ School of Public Health, Curtin University, Perth, \\ Australia; ${ }^{3}$ Thai Nguyen University of Medicine and Pharmacy, Thai Nguyen, Vietnam; ${ }^{4}$ National Institute of Hygiene and Epidemiology, Hanoi, \\ Vietnam
}

Objectives: To objectively determine and compare the physical activity (PA) levels of adults newly diagnosed with type 2 diabetes (T2D) and adults without T2D in Vietnam using an accelerometer.

Methods: A total of 120 participants with newly diagnosed T2D and 120 adults without T2D were recruited from a large hospital in Hanoi, the capital city of Vietnam. All participants wore an ActiGraph GT3X accelerometer for at least 5 days, including 1 weekend day. Freedson cut-off points were used to estimate different intensities of PA. In addition, comparisons between groups were made with respect to achieving the World Health Organization (WHO) and International Diabetes Federation (IDF) recommended PA guidelines. Results: Men with T2D had significantly lower levels of PA than men without T2D. The respective multivariable-adjusted mean values of daily step count, daily light-intensity, moderate-intensity, and moderate-to-vigorous-intensity PA were approximately 14\%, 19\%, and 22\% lower in the men with T2D than in their non-T2D counterparts. However, women with T2D accumulated a greater number of steps per day than women without T2D. Only 59.2\% of the adults with T2D met the minimum recommended level of PA (WHO and IDF), compared to $74.2 \%$ of adults without T2D $(p<0.05)$. After adjusting for potential confounders, participants with T2D experienced $50.0 \%$ significantly lower odds of achieving PA recommendations.

Conclusions: Vietnamese men with T2D were less physically active than those without T2D, and adults with T2D were less likely to meet PA guidelines. The results suggest a need for integrating PA into the self-management of this chronic condition.

Key words: Physical activity, Type 2 diabetes, Adult, Vietnam

\section{INTRODUCTION}

Diabetes is now a global epidemic; it was estimated to afflict around 415 million people worldwide in 2015 and its burden

Received: September 7, 2018 Accepted: January 15, 2019

Corresponding author: Vuong Van Do, MPH

Center for Population Health Sciences, Hanoi University of Public

Health, 1A Duc Thang Road, Hanoi 100000, Vietnam

E-mail: dvv@huph.edu.vn

This is an Open Access article distributed under the terms of the Creative Commons Attribution Non-Commercial License (http://creativecommons.org/licenses/by$\mathrm{nc} / 4.0 / /$ which permits unrestricted non-commercial use, distribution, and reproduction in any medium, provided the original work is properly cited. is projected to rise to 642 million by 2040 [1]. According to the World Health Organization (WHO), this epidemic is mostly due to the increase in type 2 diabetes (T2D), which accounts for $90 \%$ of all cases of diabetes [2]. Notably, the number of people with diabetes in Southeast Asia has increased dramatically from 17 million in 1980 to 96 million in 2014 [3]. A significant economic burden, including direct costs, is incurred by patients, their families and the health care sector, while indirect costs related to productivity are also consequences of this rise in T2D and its associated complications [3,4].

Physical activity (PA) is an integral part of T2D prevention and management. In particular, moderate-intensity and mod- 
erate-to-vigorous intensity PA have been shown to reduce T2D risk by $49 \%$ [5]. Additionally, structured exercise training can improve glycaemic control in diabetic patients, with a $0.67 \%$ reduction in hemoglobin $A_{1 c}$ levels [6]. Given the health benefits of $\mathrm{PA}$, the WHO recommends adults to accumulate at least 150 minutes of moderate-intensity PA or 75 minutes of vigorous-intensity PA per week to maintain health and prevent the development of chronic diseases [7]. Similarly, the International Diabetes Federation (IDF) advises adults with diabetes to undertake this amount of weekly PA, as well as recommending participation in resistance training 3 times per week [8].

However, information about levels of PA among individuals newly diagnosed with $\mathrm{T} 2 \mathrm{D}$ in developing countries remains limited $[9,10]$. Previous research conducted in developed countries has mostly relied on self-reported questionnaires [11-13]. Self-reported measurements, such as the Global Physical Activity Questionnaire (GPAQ) and the International Physical Activity Questionnaire (IPAQ), are valuable, but they are prone to recall bias and the effects of social desirability or social approval [14]. Moreover, a study undertaken in Canada indicated that men and women with diabetes have different psychosocial, behavioural, and clinical characteristics that affect their attitudes and behaviour towards self-care, which may impact their health outcomes [15]. Indeed, gender can modify the relationship between $\mathrm{T} 2 \mathrm{D}$ and $\mathrm{PA}$, as women with T2D have been found to be less likely to achieve the recommended PA guidelines than their non-T2D counterparts [16]. Nevertheless, there is scant evidence about T2D and gender differences in PA engagement, particularly in developing countries.

Vietnam, a developing country in Southeast Asia, has been undergoing a rapid epidemiological transition. T2D has emerged as a priority public health issue, with 1 in 14 Vietnamese adults predicted to have T2D by 2035 [17]. Previous studies have examined PA levels among the general Vietnamese population using self-reported measures $[18,19]$, but none have focused specifically on adults with $\mathrm{T} 2 \mathrm{D}$ and none have used objective PA measures. The purpose of this study was to objectively determine and compare PA levels between adults newly diagnosed with T2D and adults without T2D in Vietnam using accelerometers. We hypothesised that adults with $\mathrm{T} 2 \mathrm{D}$ would have lower levels of PA than their counterparts without T2D.

\section{METHODS}

\section{Study Design and Setting}

This cross-sectional study recruited 120 participants who were newly diagnosed with $\mathrm{T} 2 \mathrm{D}$ and another comparison group of 120 adults without T2D, comprising equal numbers of men and women $(n=60)$ in each group. The sample size was calculated based on a comparison of 2 proportions [20], accounting for a 2 -sided $95 \%$ confidence interval $(\mathrm{Cl})$ with $85 \%$ power, and assuming a $20 \%$ difference in the prevalence of achieving PA recommendations between the 2 groups.

During the period from December 2015 to February 2016, recruitment and data collection took place at a large general hospital located in Hanoi, the capital city of Vietnam. This hospital provides services for the entire catchment region of Hanoi. All potential participants attended outpatient clinics of the Department of Endocrinology and underwent a diagnostic test to confirm their T2D status. The comparison group (nonT2D) were adults selected from the Department of General Medicine of the same hospital. Informed verbal and written consent was obtained from all participants.

\section{Participants}

Participants were required to be aged 40-65 years, and the participants with T2D must have been diagnosed within the last 6 months. T2D status was determined by a physician using the 2006 WHO diagnostic criteria: a fasting plasma glucose level $\geq 7.0 \mathrm{mmol} / \mathrm{L}(126 \mathrm{mg} / \mathrm{dL})$ or a 2-hour plasma glucose level $\geq 11.1 \mathrm{mmol} / \mathrm{L}$ ( $200 \mathrm{mg} / \mathrm{dL}$ ) [21]. Participants were excluded if they were deemed by the physician to be too ill or had injuries that would affect their ability to undertake regular PA. Participants with confirmed T2D were consecutively recruited until the target quota was reached.

The T2D-free status of the comparison group was confirmed by a physician using blood test results (i.e., a fasting plasma glucose level $<7.0 \mathrm{mmol} / \mathrm{L}$ or a 2 -hour plasma glucose level $<11.1 \mathrm{mmol} / \mathrm{L}$ ). The adults without T2D were recruited when they visited the participating hospital for a general health check-up or for the treatment of minor ailments.

In total, 136 T2D patients were invited to take part in the study. Three refused and 13 persons did not subsequently wear the accelerometer as required. For the comparison group, 141 eligible adults were approached, 6 refused to take part and 15 did not wear the accelerometers properly. These participants (T2D group: $n=16$; comparison group: $n=21$ ) were excluded 
from data analysis, resulting in 120 participants in each arm of the study.

\section{Physical Activity Measurements}

ActiGraph GT3X+ accelerometers (Actigraph Corp., Pensacola, FL, USA), a reliable, valid and widely accepted method [22], were used to measure PA levels and intensity (sedentary, light, moderate, or vigorous). Participants were required to wear the accelerometer on their right hip for at least 5 days, including at least 1 weekend day, for 10 hours daily, excluding waterbased activities. Non-wearing time was defined as 60 consecutive minutes of zero counts, with an allowance of up to $2 \mathrm{~min}$ utes of counts from 1 to 100. The Freedson cut-off points for adults were adopted to estimate different intensities of PA, with sedentary behaviour being less than 100 counts per minute (cpm), light-intensity PA being 100-1951 cpm, moderateintensity PA being 1952-5724 cpm, vigorous-intensity PA being 5725-9498 cpm, very-vigorous-intensity PA being $>9498$ cpm, and moderate-to-vigorous-intensity PA being $\geq 1952$ cpm [23].

\section{Other Variables}

Information on demographic characteristics, cigarette smoking, and alcohol consumption was collected through an interviewer-administered questionnaire, whereas anthropometric measurements (weight, height, waist and hip circumferences) were collected using standardised equipment and protocols [24]. Clinical information, such as blood pressure and cholesterol levels, was retrieved from hospital medical records. Hypertension was defined as a systolic blood pressure (SBP) $\geq 140$ $\mathrm{mmHg}$ or a diastolic blood pressure (DBP) $\geq 90 \mathrm{mmHg}$ [25], and hypercholesterolemia was defined as total cholesterol $\geq 200 \mathrm{mg} / \mathrm{dL}$ or low-density lipoprotein cholesterol $\geq 130$ $\mathrm{mg} / \mathrm{dL}$ [26].

\section{Statistical Analysis}

The characteristics of participants were compared between groups using the chi-square or Fisher exact tests for categorical variables and the $t$-test or the Wilcoxon rank-sum test for continuous variables. Accelerometry data were exported from ActiLife 6, a data analysis software provided by Actigraph. The

Table 1. Characteristics of participants by gender and type 2 diabetes (T2D) status

\begin{tabular}{|c|c|c|c|c|c|c|c|c|c|}
\hline \multirow{2}{*}{ Characteristics } & \multicolumn{3}{|c|}{ Total } & \multicolumn{3}{|c|}{ Men } & \multicolumn{3}{|c|}{ Women } \\
\hline & T2D & Non-T2D & $p$-value ${ }^{1}$ & T2D & Non-T2D & $p$-value ${ }^{1}$ & T2D & Non-T2D & $p$-value ${ }^{1}$ \\
\hline No. of participants & 120 & 120 & & 60 & 60 & & 60 & 60 & \\
\hline Age (y) & $58.6 \pm 5.2$ & $58.0 \pm 5.6$ & 0.45 & $58.8 \pm 5.4$ & $58.1 \pm 5.9$ & 0.63 & $58.3 \pm 5.1$ & $57.7 \pm 5.5$ & 0.56 \\
\hline Education level $^{2}$ & & & 0.25 & & & 0.08 & & & 0.93 \\
\hline Primary school or lower & $10(8.3)$ & $6(5.0)$ & & $5(8.3)$ & $1(1.7)$ & & $5(8.3)$ & $5(8.3)$ & \\
\hline Secondary school & $44(36.7)$ & $36(30.0)$ & & $20(33.3)$ & $14(23.3)$ & & $24(40.0)$ & $22(36.7)$ & \\
\hline High school or above & $66(55.0)$ & $78(65.0)$ & & $35(58.3)$ & $45(75.0)$ & & $31(51.7)$ & $33(55.0)$ & \\
\hline Married & $119(99.2)$ & $114(95.0)$ & 0.06 & $60(100)$ & 59 (98.3) & 0.50 & $59(98.3)$ & 55 (91.7) & 0.24 \\
\hline Smoking behaviour & & & 0.01 & & & 0.01 & & & 0.24 \\
\hline Never & $78(65.0)$ & 95 (79.2) & & $21(35.0)$ & $35(58.3)$ & & $57(95.0)$ & $60(100)$ & \\
\hline Former or current & $42(35.0)$ & $25(20.8)$ & & $39(65.0)$ & $25(41.7)$ & & $3(5.0)$ & $0(0.0)$ & \\
\hline Alcohol consumption & & & 0.44 & & & 0.17 & & & 0.50 \\
\hline Never & $69(57.5)$ & $63(52.5)$ & & $10(16.7)$ & $5(8.3)$ & & 59 (98.3) & $58(96.7)$ & \\
\hline Former or current & $51(42.5)$ & $57(47.5)$ & & 50 (83.3) & $55(91.7)$ & & $1(1.7)$ & $2(3.3)$ & \\
\hline First-degree family diabetes history ${ }^{3}$ & $46(38.3)$ & $13(10.8)$ & $<0.01$ & $22(36.7)$ & $4(6.7)$ & $<0.01$ & $24(40.0)$ & $9(15.0)$ & $<0.01$ \\
\hline Body mass index $\left(\mathrm{kg} / \mathrm{m}^{2}\right)$ & $23.5 \pm 2.7$ & $22.3 \pm 2.4$ & $<0.01$ & $23.2 \pm 2.4$ & $22.4 \pm 2.5$ & 0.08 & $23.9 \pm 2.9$ & $22.2 \pm 2.3$ & $<0.01$ \\
\hline Waist-hip ratio & $0.9 \pm 0.1$ & $0.9 \pm 0.1$ & 0.90 & $0.9 \pm 0.0$ & $0.9 \pm 0.1$ & 0.39 & $0.9 \pm 0.1$ & $0.9 \pm 0.0$ & 0.57 \\
\hline Total cholesterol (mmol/L) & $5.0 \pm 1.2$ & $5.0 \pm 0.9$ & 0.39 & $4.9 \pm 1.1$ & $5.1 \pm 0.9$ & 0.05 & $5.2 \pm 1.3$ & $4.9 \pm 1.0$ & 0.48 \\
\hline Systolic blood pressure (mmHg) & $130.0 \pm 15.0$ & $125.0 \pm 12.5$ & 0.01 & $133.0 \pm 13.5$ & $127.0 \pm 11.5$ & 0.02 & $127.0 \pm 16.1$ & $122.0 \pm 13.0$ & $<0.01$ \\
\hline Diastolic blood pressure (mmHg) & $81.0 \pm 10.0$ & $75.0 \pm 8.5$ & 0.01 & $83.0 \pm 11.2$ & $77.0 \pm 7.4$ & $<0.01$ & $80.0 \pm 8.6$ & $74.0 \pm 9.2$ & $<0.01$ \\
\hline
\end{tabular}

Data are presented as mean \pm standard deviation or number (\%).

${ }^{1}$ Based on the chi-square or Fisher exact test for categorical variables and the $t$-test or rank-sum test for continuous variables.

2Primary school: having completed grade 5; secondary school: having completed grade 9; high school: having completed grade 12.

${ }^{3}$ Having parents or siblings with $\mathrm{T} 2 \mathrm{D}$. 
levels of PA of participants with and without T2D were compared using generalised linear regression models, adjusting for education (primary school or lower, secondary school, high school or above), age (continuous), gender (men or women), hypertension (yes or no), hypercholesterolemia (yes or no), and body mass index ([BMI], continuous). Mean values and standard errors were calculated for time spent wearing the device, daily step count, and level of PA intensity. Participants were also classified as achieving or not achieving the WHO and IDF PA recommendations, based on whether they accumulated $\geq 150$ minutes of moderate-intensity activity or $\geq 75$ minutes of vigorous-intensity activity per week. The association between T2D status and the prevalence of achieving the PA recommendation was assessed using multivariable logistic regression models, accounting for the aforementioned poten- tial covariates. Adjusted odds ratios (ORs) and associated 95\% $\mathrm{Cls}$ were calculated. The potential interaction between gender and T2D status was examined through the Wald test of the regression coefficient for the product term (gender $\times$ T2D) [27]. All statistical analyses were performed using Stata version 13 (StataCorp., College Station, TX, USA).

\section{RESULTS}

Table 1 presents the characteristics of all participants according to gender and T2D status. Overall, participants with T2D had a significantly higher prevalence of ever smoking and a family history of T2D, a higher BMI, and higher SBP and DBP than their counterparts without T2D. There was no difference between the 2 groups in terms of education level, marital sta-

Table 2. Daily accelerometer-measured PA levels, wearing time, step count, and percentage meeting PA recommendations (WHO \& IDF)

\begin{tabular}{|c|c|c|c|c|c|c|c|}
\hline \multirow{2}{*}{ PA level (min) } & \multicolumn{2}{|c|}{ Total } & \multicolumn{2}{|c|}{ Men } & \multicolumn{2}{|c|}{ Women } & \multirow{2}{*}{$\begin{array}{c}p \text { for } \\
\text { interaction }\end{array}$} \\
\hline & T2D & Non-T2D & T2D & Non-T2D & T2D & Non-T2D & \\
\hline \multicolumn{8}{|c|}{ Crude mean $\pm S E$ (adjusted mean $\pm S E)^{2}$} \\
\hline No. of participants & 120 & 120 & 60 & 60 & 60 & 60 & \\
\hline \multirow[t]{2}{*}{ Wearing time } & $845 \pm 102$ & $866 \pm 98$ & $851 \pm 102$ & $871 \pm 89$ & $840 \pm 102$ & $863 \pm 105$ & 0.93 \\
\hline & $(848 \pm 10)$ & $(867 \pm 10)$ & $(864 \pm 13)$ & $(867 \pm 13)$ & $(836 \pm 15)$ & $(864 \pm 14)$ & 0.53 \\
\hline \multirow[t]{2}{*}{ Steps } & $5632 \pm 230^{*}$ & $6374 \pm 273$ & $5550 \pm 348^{*}$ & $7047 \pm 460$ & $5715 \pm 304$ & $5702 \pm 272$ & 0.03 \\
\hline & $(5866 \pm 8)^{* *}$ & $(6140 \pm 8)$ & $(5823 \pm 11)^{* *}$ & $(6744 \pm 11)$ & $(5973 \pm 11)^{* *}$ & $(5495 \pm 10)$ & 0.07 \\
\hline \multirow[t]{2}{*}{ Light-intensity } & $176 \pm 5^{* *}$ & $201 \pm 6$ & $165 \pm 8^{* *}$ & $205 \pm 9$ & $188 \pm 7$ & $196 \pm 8$ & 0.03 \\
\hline & $(177 \pm 6)^{* *}$ & $(202 \pm 6)$ & $(167 \pm 9)^{* *}$ & $(206 \pm 9)$ & $(189 \pm 8)$ & $(197 \pm 7)$ & 0.01 \\
\hline \multirow[t]{2}{*}{ Moderate-intensity } & $32 \pm 2^{*}$ & $39 \pm 2$ & $33 \pm 3^{* *}$ & $46 \pm 4$ & $30 \pm 3$ & $31 \pm 2$ & 0.04 \\
\hline & $(33 \pm 2)$ & $(37 \pm 2)$ & $(35 \pm 3)^{*}$ & $(45 \pm 3)$ & $(32 \pm 3)$ & $(30 \pm 2)$ & 0.07 \\
\hline \multirow[t]{2}{*}{ Moderate-to-vigorous-intensity } & $32 \pm 2^{*}$ & $39 \pm 2$ & $34 \pm 3^{* *}$ & $47 \pm 4$ & $31 \pm 3$ & $32 \pm 2$ & 0.04 \\
\hline & $(34 \pm 2)$ & $(38 \pm 2)$ & $(35 \pm 3)^{*}$ & $(45 \pm 3)$ & $(32 \pm 3)$ & $(30 \pm 3)$ & 0.07 \\
\hline \multirow[t]{2}{*}{ Sedentary } & $634 \pm 9$ & $624 \pm 9$ & $649 \pm 13$ & $617 \pm 13$ & $619 \pm 12$ & $632 \pm 12$ & 0.85 \\
\hline & $(635 \pm 10)$ & $(626 \pm 9)$ & $(656 \pm 14)^{*}$ & $(617 \pm 13)$ & $(614 \pm 13)$ & $(636 \pm 13)$ & 0.75 \\
\hline \multicolumn{8}{|l|}{ Met recommendations } \\
\hline \multicolumn{8}{|l|}{ IDF-WHO level ${ }^{3}$} \\
\hline $\mathrm{n}(\%)$ & $71(59.2)^{*}$ & 89 (74.2) & $38(63.3)^{*}$ & $49(81.7)$ & $33(55.0)$ & $40(66.7)$ & $\mathrm{N} / \mathrm{A}$ \\
\hline $\mathrm{aOR}(95 \% \mathrm{CI})^{4}$ & $0.5(0.3,0.9)$ & 1.00 (reference) & $0.4(0.1,1.0)$ & 1.00 (reference) & $0.7(0.3,1.6)$ & 1.00 (reference) & 0.78 \\
\hline \multicolumn{8}{|l|}{ WHO (benefits) ${ }^{5}$} \\
\hline $\mathrm{n}(\%)$ & $32(26.7)$ & $44(36.7)$ & $18(30.0)^{*}$ & $30(50.0)$ & 14 (23.3) & 14 (23.3) & $\mathrm{N} / \mathrm{A}$ \\
\hline $\mathrm{aOR}(95 \% \mathrm{CI})^{4}$ & $0.8(0.4,1.4)$ & 1.00 (reference) & $0.5(0.2,1.1)$ & 1.00 (reference) & $1.4(0.5,3.5)$ & 1.00 (reference) & 0.17 \\
\hline
\end{tabular}

PA, physical activity; WHO, World Health Organisation; IDF, International Diabetes Federation; T2D, type 2 diabetes; SE, standard error; aOR, adjusted odds ratio; Cl, confidence interval; N/A, not applicable.

${ }^{1}$ Interactive effects between gender and T2D status on PA data according to the Wald test.

${ }^{2}$ Obtained from generalised linear models adjusting for education, age, gender, hypertension, hypercholesterolemia, and body mass index.

${ }^{3}$ Accumulation of $\geq 150$ minutes of moderate-intensity activity or $\geq 75$ minutes of vigorous-intensity activity per week.

${ }^{4}$ Obtained from logistic regression models adjusting for education, age, gender, hypertension, hypercholesterolemia, and body mass index.

${ }^{5}$ Accumulation of $\geq 300$ minutes of moderate-intensity activity or $\geq 150$ minutes of vigorous-intensity activity per week (additional health benefits).

${ }^{*} p<0.05,{ }^{* *} p<0.01$. 
tus, waist-hip ratio, total cholesterol, or alcohol consumption. Men with diabetes had a significantly higher prevalence of ever smoking and a family history of T2D, as well as higher SBP and DBP, than men without T2D. Compared to women without $T 2 D$, those with $T 2 D$ were more likely to have a family history of T2D, a higher BMI, and higher SBP and DBP. In contrast, no significant differences were observed for socio-demographic and lifestyle factors.

Table 2 shows the wearing time, PA levels, and prevalence of meeting the recommended WHO and IDF PA guidelines. On average, participants wore the accelerometer device for more than $14 \mathrm{hr} / \mathrm{d}$. There was no difference in wearing time between the T2D and non-T2D groups in either the crude or adjusted model. Although all PA parameters (i.e., step count, light-intensity, moderate-intensity, and moderate-to-vigorous-intensity) appeared to be lower among T2D participants without adjustment for covariates, only the daily step count and light-intensity PA remained significantly different between the 2 groups after accounting for the effects of confounding factors. In the stratified analysis, men with T2D engaged in fewer steps and spent less time at all PA levels than men without T2D, and also had more sedentary time on a daily basis. In contrast, women with T2D took more daily steps than women without T2D, although no apparent differences were found for the other PA variables. A significant interaction was observed between gender and T2D status for light-intensity PA only $\left(p_{\text {interaction }}=0.01\right)$.

Table 2 shows that only $59.2 \%$ of the participants with T2D met the minimum recommended level of PA, compared to $74.2 \%$ of the participants without T2D $(p<0.05)$. Stratification by gender further revealed that men with $\mathrm{T} 2 \mathrm{D}$ (63.3\%) were significantly less likely $(p=0.03)$ to meet the recommended PA level than men without T2D (81.7\%). The corresponding prevalence for women was lower (55.0\% and 66.7\%, respectively), although the difference was not statistically significant $(p=$ 0.45). Overall, just over one-quarter (26.7\%) of T2D participants achieved the WHO recommendation for sufficient PA to obtain 'additional health benefits', in contrast to $36.7 \%$ for those without T2D. Compared to adults without T2D, those with T2D were $50.0 \%$ less likely to achieve the PA recommendations, and there was no evidence of interaction between gender and T2D status. However, the proportion of those who engaged in enough PA to achieve additional health benefits was not significantly different between the 2 groups.

\section{DISCUSSION}

This study investigated PA levels in adults newly diagnosed with T2D in Vietnam. To the best of our knowledge, this is the first such study using accelerometers to objectively measure PA levels of adults with T2D and without T2D in a developing country. The results showed that adults with T2D accumulated fewer steps and spent less time on light PA per day than adults without T2D. In particular, men with T2D engaged in less PA than their non-T2D counterparts, and a lower proportion of adults with T2D met the recommended PA guidelines for health benefits.

Our finding that adults with T2D were less physically active is consistent with the few studies previously undertaken in developed countries $[11,13,16]$. However, the proportion of participants attaining the PA recommendation was higher than those reported in Lebanon ( $n=2195)$ [13] and Australia $(n=629)$ [16]. The Lebanese study used the self-reported IPAQ short version to measure PA and found that $44.4 \%$ of participants with T2D exceeded 150 minutes of moderate PA per week, while $29.7 \%$ of participants with T2D in the Australian study met the PA guidelines as measured by pedometers. This disparity may be attributed to differences in socioeconomic status between countries, since people from lower-income countries tend to be more active than those from high-income countries $[28,29]$. As an alternate possibility, our findings were based on the use of accelerometers as a measurement tool. Accelerometers are considered to be an objective, practical, and accurate way of measuring the volume and intensity of the PA [30].

We observed significantly lower PA levels among only man participants with T2D. Evidence of effect modification by gender on the association between T2D and PA remains scarce in the literature. A previous study of 629 Australians (293 with T2D and 336 without T2D) reported no significant difference in pedometer-measured daily step counts between men with and without T2D [16]. This discrepancy may be due to differences in cultural, socio-demographic, and environmental factors, or the type of PA measuring instrument. In addition, our finding of a positive association between T2D and daily step count in women is contrary to the aforementioned Australian study that reported a significantly higher number of steps per day among women without T2D. Women with T2D may have excessive cardiovascular risk compared to men with diabetes [16], and thus their underlying cardiometabolic abnormalities 
may have hampered them from engaging frequently in PA. In the present study, it is plausible that Vietnamese women with T2D are more health-conscious than men [31] and may seek medical advice [32] to manage their diabetes, leading to more frequent engagement in PA [33].

It has been suggested that individuals with diabetes have poor physical condition, functional limitations [34], and frequent sensations of fatigue [35], which prevent them from being physically active. In addition, a qualitative study of patients with dysregulated diabetes and complications or other comorbidities reported that lack of professional guidance, lack of accessibility to exercise facilities, and lack of awareness of where or how to exercise were major barriers to PA [34]. Adults newly diagnosed with T2D may not experience physical constraints; however, changing well-established habits, negative perceptions of the 'new' or recommended diabetes regimen, together with a lack of knowledge and understanding of how to live with $\mathrm{T} 2 \mathrm{D}$ may act as barriers to being physically active [36]. Longitudinal and large-scale studies are needed to ascertain factors affecting PA engagement by adults with T2D in Vietnam.

Participation in PA can be affected by individual aspects and socio-environmental support $[37,38]$. Research has indicated that physicians play a key role in increasing the PA levels of adults with diabetes $[13,39]$. In particular, physicians can help patients newly diagnosed with diabetes restore their confidence through education on the management of their condition [39]. It has been reported that adults with $T 2 D$ are more likely to engage in PA than adults without T2D, after receiving the same advice from physicians [13]. However, an Australian study ( $n=1799)$ indicated that only $18.0 \%$ of patients received PA recommendations from their general practitioners [40]. Therefore, ready opportunities exist for physicians to become involved in PA education for adults with T2D.

The major strength of our study was the use of accelerometers to provide reliable and valid estimations of different intensity levels of PA. Moreover, only adults newly diagnosed with T2D were selected to avoid the longer-term impacts of the disease on PA levels. We acknowledge that our convenience sample limited the generalizability of the findings. However, all participants were recruited from the same large hospital that serves the entire Hanoi catchment region, making the study population reasonably representative. Nonetheless, the lack of information about prior PA history made it difficult to assess possible changes in PA after the T2D diagnosis. Finally, the results observed in a cross-sectional study do not necessarily indicate a causal relationship between T2D and PA.

In conclusion, Vietnamese adults with newly diagnosed T2D, especially men, were found to be less physically active and less likely to achieve the WHO and IDF recommended PA levels than others without the condition. As such, designing and promoting integrated PA education programs (home-based, community-based, or hospital-based for individuals with $\mathrm{T} 2 \mathrm{D}$ in the early stages after diagnosis are imperative to the selfmanagement of the disease, with implications for its progression and potential complications. Physicians should actively educate T2D patients about the important role of PA in the management of their condition. Future research examining barriers to achieving PA recommendations among newly diagnosed T2D patients is required in order to develop effective primary care interventions.

\section{CONFLICT OF INTEREST}

The authors have no conflicts of interest associated with the material presented in this paper.

\section{ACKNOWLEDGEMENTS}

The authors are grateful to the medical and nursing staff of the participating hospital for their assistance in participant recruitment.

\section{ORCID}

Vuong Van Do http://orcid.org/0000-0003-2140-9772

Jonine Jancey https://orcid.org/0000-0002-7894-2896

Ngoc Minh Pham https://orcid.org/0000-0002-4138-6427

Chung Thanh Nguyen https://orcid.org/0000-0001-77191405

Minh Van Hoang https://orcid.org/0000-0002-4749-5536

Andy H. Lee https://orcid.org/0000-0003-4120-5005

\section{REFERENCES}

1. Ogurtsova K, da Rocha Fernandes JD, Huang Y, Linnenkamp U, Guariguata L, Cho NH, et al. IDF diabetes atlas: global estimates for the prevalence of diabetes for 2015 and 2040. Diabetes Res Clin Pract 2017;128:40-50.

2. World Health Organization. Diabetes; 2018 [cited 2019 Feb 
28]. Available from: https://www.who.int/en/news-room/ fact-sheets/detail/diabetes.

3. World Health Organization. Global report on diabetes; 2016 [cited 2018 Jun 1]. Available from: https://www.who.int/diabetes/global-report/en/.

4. Bommer C, Heesemann E, Sagalova V, Manne-Goehler J, Atun $\mathrm{R}$, Bärnighausen $\mathrm{T}$, et al. The global economic burden of diabetes in adults aged 20-79 years: a cost-of-illness study. Lancet Diabetes Endocrinol 2017;5(6):423-430.

5. Laaksonen DE, Lindström J, Lakka TA, Eriksson JG, Niskanen L, Wikström $\mathrm{K}$, et al. Physical activity in the prevention of type 2 diabetes: the Finnish diabetes prevention study. Diabetes 2005; 54(1):158-165.

6. Umpierre D, Ribeiro PA, Kramer CK, Leitão CB, Zucatti AT, Azevedo $\mathrm{MJ}$, et al. Physical activity advice only or structured exercise training and association with $\mathrm{HbA1c}$ levels in type 2 diabetes: a systematic review and meta-analysis. JAMA 2011; 305(17):1790-1799.

7. World Health Organization. Global recommendations on physical activity for health; 2010 [cited 2018 Jun 9]. Available from: https://www.who.int/dietphysicalactivity/factsheet_recommendations/en/.

8. International Diabetes Federation. Global guideline for type 2 diabetes; 2012 [cited 2018 Jun 1]. Available from: https://www. idf.org/e-library/guidelines/79-global-guideline-for-type-2diabetes.

9. Katulanda P, Jayawardena R, Ranasinghe P, Rezvi Sheriff MH, Matthews DR. Physical activity patterns and correlates among adults from a developing country: the Sri Lanka Diabetes and Cardiovascular Study. Public Health Nutr 2013;16(9):1684-1692.

10. Ranasinghe DC, Ranasinghe P, Jayawardena R, Matthews DR, Katulanda P. Evaluation of physical activity among adults with diabetes mellitus from Sri Lanka. Int Arch Med 2014;7:15.

11. Morrato EH, Hill JO, Wyatt HR, Ghushchyan V, Sullivan PW. Physical activity in U.S. adults with diabetes and at risk for developing diabetes, 2003. Diabetes Care 2007;30(2):203-209.

12. Nelson KM, Reiber G, Boyko EJ; NHANES III. Diet and exercise among adults with type 2 diabetes: findings from the third National Health and Nutrition Examination Survey (NHANES III). Diabetes Care 2002;25(10):1722-1728.

13. Sibai AM, Costanian C, Tohme R, Assaad S, Hwalla N. Physical activity in adults with and without diabetes: from the 'highrisk' approach to the 'population-based' approach of prevention. BMC Public Health 2013;13:1002.

14. Adams SA, Matthews CE, Ebbeling CB, Moore CG, Cunning- ham JE, Fulton J, et al. The effect of social desirability and social approval on self-reports of physical activity. Am J Epidemiol 2005;161(4):389-398.

15. Gucciardi E, Wang SC, DeMelo M, Amaral L, Stewart DE. Characteristics of men and women with diabetes: observations during patients' initial visit to a diabetes education centre. Can Fam Physician 2008;54(2):219-227.

16. Kelly J, Edney K, Moran C, Srikanth V, Callisaya M. Gender differences in physical activity levels of older people with type 2 diabetes mellitus. J Phys Act Health 2016;13(4):409-415.

17. Pham NM, Eggleston K. Prevalence and determinants of diabetes and prediabetes among Vietnamese adults. Diabetes Res Clin Pract 2016;113:116-124.

18. Bui TV, Blizzard CL, Luong KN, Truong Nle V, Tran BQ, Otahal P, et al. Physical activity in Vietnam: estimates and measurement issues. PLoS One 2015;10(10):e0140941.

19. Trinh OT, Nguyen ND, Dibley MJ, Phongsavan P, Bauman AE. The prevalence and correlates of physical inactivity among adults in Ho Chi Minh City. BMC Public Health 2008;8:204.

20. Fleiss JL, Levin B, Paik MC. Statistical methods for rates and proportions. 3rd ed. Hoboken: John Wiley \& Sons; 2004, p. 64-85.

21. World Health Organization. Definition and diagnosis of diabetes mellitus and intermediate hyperglycaemia: 2006 [cited 2018 Jul 1]. Available from: https://www.who.int/diabetes/ publications/diagnosis_diabetes2006/en/.

22. Freedson PS, Miller K. Objective monitoring of physical activity using motion sensors and heart rate. Res Q Exerc Sport 2000; 71(2 Suppl):S21-S29.

23. Dyrstad SM, Hansen BH, Holme IM, Anderssen SA. Comparison of self-reported versus accelerometer-measured physical activity. Med Sci Sports Exerc 2014;46(1):99-106.

24. World Health Organization. WHO STEPS surveillance manual: the WHO STEPwise approach to chronic disease risk factor surveillance; 2005 [cited $2018 \mathrm{Jul}$ 1]. Available from: http:// www.who.int/iris/handle/10665/43376.

25. Chobanian AV, Bakris GL, Black HR, Cushman WC, Green LA Izzo JL Jr, et al. The seventh report of the joint national committee on prevention, detection, evaluation, and treatment of high blood pressure: the JNC 7 report. JAMA 2003;289(19): 2560-2572.

26. National Cholesterol Education Program (NCEP) Expert Panel on Detection, Evaluation, and Treatment of High Blood Cholesterol in Adults (Adult Treatment Panel III). Third report of the National Cholesterol Education Program (NCEP) expert panel on detection, evaluation, and treatment of high blood 
cholesterol in adults (Adult Treatment Panel III) final report. Circulation 2002;106(25):3143-3421.

27. Wald A. Tests of statistical hypotheses concerning several parameters when the number of observations is large. Trans Am Math Soc 1943;54(3):426-482.

28. Dumith SC, Hallal PC, Reis RS, Kohl HW 3rd. Worldwide prevalence of physical inactivity and its association with human development index in 76 countries. Prev Med 2011;53(1-2): 24-28.

29. Hallal PC, Andersen LB, Bull FC, Guthold R, Haskell W, Ekelund $U$, et al. Global physical activity levels: surveillance progress, pitfalls, and prospects. Lancet 2012;380(9838):247-257.

30. Hills AP, Mokhtar N, Byrne NM. Assessment of physical activity and energy expenditure: an overview of objective measures. Front Nutr 2014;1:5.

31. Fitzgerald JT, Anderson RM, Davis WK. Gender differences in diabetes attitudes and adherence. Diabetes Educ 1995;21(6): 523-529.

32. Siddiqui MA, Khan MF, Carline TE. Gender differences in living with diabetes mellitus. Mater Sociomed 2013;25(2):140-142.

33. Meyer K, Niemann S, Abel T. Gender differences in physical activity and fitness - association with self-reported health and health-relevant attitudes in a middle-aged Swiss urban population. J Public Health 2004;12(4):283-290.
34. Lidegaard LP, Schwennesen N, Willaing I, Faerch K. Barriers to and motivators for physical activity among people with type 2 diabetes: patients' perspectives. Diabet Med 2016;33(12): 1677-1685.

35. Thomas N, Alder E, Leese GP. Barriers to physical activity in patients with diabetes. Postgrad Med J 2004;80(943):287-291.

36. Booth AO, Lowis C, Dean M, Hunter SJ, McKinley MC. Diet and physical activity in the self-management of type 2 diabetes: barriers and facilitators identified by patients and health professionals. Prim Health Care Res Dev 2013;14(3):293-306.

37. Donahue KE, Mielenz TJ, Sloane PD, Callahan LF, Devellis RF. Identifying supports and barriers to physical activity in patients at risk for diabetes. Prev Chronic Dis 2006;3(4):A119.

38. Sterdt E, Liersch S, Walter U. Correlates of physical activity of children and adolescents: a systematic review of reviews. Health Educ J 2014;73(1):72-89.

39. Duclos M, Dejager S, Postel-Vinay N, di Nicola S, Quéré S, Fiquet B. Physical activity in patients with type 2 diabetes and hypertension--insights into motivations and barriers from the MOBILE study. Vasc Health Risk Manag 2015;11:361-371.

40. Short CE, Hayman M, Rebar AL, Gunn KM, De Cocker K, Duncan MJ, et al. Physical activity recommendations from general practitioners in Australia. Results from a national survey. Aust N Z J Public Health 2016;40(1):83-90. 